\title{
Feasibility of administering human pancreatic cancer chemotherapy in a spontaneous pancreatic cancer mouse model
}

\author{
Abagail M. Delahoussaye ${ }^{1 \dagger}$, Joseph Abi Jaoude ${ }^{2 \dagger}$, Morgan Green ${ }^{3}$, Tara N. Fujimoto ${ }^{1}$, Jessica Molkentine ${ }^{1}$, \\ Carolina J. Garcia Garcia ${ }^{1}$, Jason P. Gay ${ }^{4}$, Ningping Feng ${ }^{4}$, Joseph Marszalek ${ }^{4}$, Natalie Fowlkes ${ }^{3}$ and \\ Cullen M. Taniguchi ${ }^{1 *}$
}

\begin{abstract}
Background: Both modified FOLFIRINOX ( $\mathrm{mFFX)} \mathrm{and} \mathrm{gemcitabine/nab-paclitaxel} \mathrm{chemotherapy} \mathrm{regimens} \mathrm{have}$ been shown to improve clinical outcomes in patients with pancreatic cancer, and are often used interchangeably as the standard of care. Preclinical studies often do not use these regimens, since administering these multiagent approaches can be difficult. In this study, we assessed the feasibility of administering these two chemotherapy regimens in spontaneous pancreatic tumors using KPC mice with the ultimate goal of advancing preclinical studies.

Methods: KPC mice were created by breeding $\mathrm{Kras}^{\mathrm{LLL}-G 12 \mathrm{D} /+}$ to $\mathrm{Trp} 53^{\mathrm{fl} / \mathrm{f}} ; \mathrm{Ptf1} \mathrm{a}^{\mathrm{Cre} /+}$, resulting in $\mathrm{Kras} \mathrm{sL}^{\mathrm{LSL}}$ ${ }_{G 12 D /+} ; p 53^{f / /+}$;Ptfla $a^{C r /+}$ mice. At 14 weeks of age, mice were palpated for spontaneous tumor growth that was verified using ultrasounds. Mice with tumors under $15 \mathrm{~mm}$ in diameter were used. The mice were assigned to one of seven treatment regimens: 1 cycle of mFFX (FFXX1), 2 cycles of mFFX (FFX X2), 1 cycle of mFFXwith 40 Gy SBRT (FFX SBRT), 1 cycle of gemcitabine/nab-paclitaxel (GEM/ABX1), 2 cycles of gemcitabine/nab-paclitaxel (GEM/AB X2), 2 cycles of gemcitabine/nab-paclitaxel with 40 Gy SBRT (GEM/AB SBRT), or saline only (control).

Results: In total, 92 mice were included. The median OS in the FFX X2 group was slightly longer that the median OS in the FFX X1 group ( 15 days vs 11 days, $P=0.003$ ). Mice in the GEM/AB X2 group had longer OS when compared to mice in the GEM/AB X1 group ( 33.5 vs 13 days, $P=0.001$ ). Mice treated with chemotherapy survived longer than untreated control animals (median OS: 6.5 days, $P<0.001$ ). Moreover, in mice treated with chemotherapy, mice that received 2 cycles of GEM/AB X2 had the longest survival, while the FFX X1 group had the poorest OS $(P<0.001)$. The addition of chemotherapy was associated with reduced number of myeloid and lymphoid cell types, except for CD4 + cells whose levels were largely unaltered only in tumors treated with gemcitabine/nab-paclitaxel. Lastly, chemotherapy followed by consolidative SBRT trended towards increased local control and survival.
\end{abstract}

\footnotetext{
*Correspondence: ctaniguchi@mdanderson.org

${ }^{\dagger}$ Abagail M. Delahoussaye and Joseph Abi Jaoude contributed equally as first

authors.

${ }^{1}$ Department of Experimental Radiation Oncology, The University of Texas

MD Anderson Cancer Center, Houston, TX 77030, USA

Full list of author information is available at the end of the article
} original author(s) and the source, provide a link to the Creative Commons licence, and indicate if changes were made. The images or other third party material in this article are included in the article's Creative Commons licence, unless indicated otherwise in a credit line to the material. If material is not included in the article's Creative Commons licence and your intended use is not permitted by statutory regulation or exceeds the permitted use, you will need to obtain permission directly from the copyright holder. To view a copy of this licence, visit http://creativecommons.org/licenses/by/4.0/. The Creative Commons Public Domain Dedication waiver (http://creativeco mmons.org/publicdomain/zero/1.0/) applies to the data made available in this article, unless otherwise stated in a credit line to the data. 
Conclusions: We demonstrate the utility and feasibility of clinically relevant mFOLFIRINOX and gemcitabine/nabpaclitaxel in preclinical models of pancreatic cancer.

Keywords: KPC mice, mFOLFIRINOX, Gemcitabine, Nab-paclitaxel, Pancreatic cancer, SBRT

\section{Background}

Pancreatic cancer is one of the leading causes of cancerrelated death in the world $[1,2]$. Owing to the limited role of screening in pancreatic cancer, tumors are often discovered at advanced stages, which contributes to poor outcomes and severe morbidity and mortality. While surgery is the main curative option in pancreatic cancer, a large proportion of patients with pancreatic cancer are initially diagnosed with unresectable disease that would not benefit from local therapy $[1,3]$. Moreover, pancreatic cancer is known to metastasize early in the disease process [4]. Therefore, chemotherapy remains a major part of treatment for all stages of pancreatic cancer.

Many chemotherapy regimens have been suggested for the treatment of pancreatic cancer. Previously, gemcitabine and 5-fluorouracil were used as monotherapy, however recurrence rates remained high $[5,6]$. As such, modern systemic treatment consists of powerful combinations of chemotherapy. The two most common regimens used in the modern treatment of pancreatic cancer are modified FOLFIRINOX (mFFX: oxaliplatin, irinotecan, leucovorin, fluorouracil) and gemcitabine/nabpaclitaxel [7-10]. Many studies and trials analyzing the role of those regimens showed superior clinical results compared to conventional monotherapy treatments, and established the role of mFOLFIRINOX and gemcitabine/nab-paclitaxel as standard of care in pancreatic cancer.

While those regimens have been thoroughly studied in humans, limited data on the use of mFOLFIRINOX and gemcitabine/nab-paclitaxel are present in pre-clinical studies. In particular, the clinical utility and toxicity of those regimens is still not clear in spontaneous pancreatic tumors in KPC mice. Thus, in order to more closely mimic human clinical treatment, pre-clinical models need to implement similar chemotherapy regimens. In this study, we aim to assess the feasibility of administering mFOLFIRINOX and gemcitabine/nab-paclitaxel in spontaneous pancreatic tumors using a KPC mouse model, as well as their toxicity profiles.

\section{Methods}

\section{KPC mice and tumor diagnosis}

All mouse work was approved and done in accordance with the Institutional Animal Care and Use Committee of The University of Texas MD Anderson Cancer
Center under protocol IACUC \#00001252-RN02. The studies were carried out in compliance with ARRIVE guidelines. Both female and male mice were used in this study. Mice were maintained on a 12-h light/dark cycle and were provided with sterilized water and standard rodent chow (Prolab Isopro RMH 3000 irradiated feed). Experiments were carried out during the light cycle.

KPC mice were created by breeding Kras $^{L S L-}$ ${ }_{G 12 D /+}$ to $\operatorname{Trp} 53^{f l / f} ; \mathrm{Ptfl} 1 \alpha^{\mathrm{Cr} e /+}$, resulting in a $\mathrm{Kras}^{L S L-}$ ${ }^{G 12 D /+} ; \operatorname{Trp} 53^{f l /+} ;$ Ptf $1 \alpha^{\text {Cre }}$ (KPC) mice, which is a validated model of locally advanced pancreatic cancer [11]. Beginning at 14 weeks of age, mice were screened weekly by palpation under anesthesia for tumor growth, as previously described [12]. Suspicious intrabdominal masses, were then subjected to small animal ultrasound imaging (FujiFilm Vevo2100), with a $30 \mathrm{MHz}$ transducer, acquired in B-MODE to estimate volumes from the long and short axes $[12,13]$.

\section{Chemotherapy}

We used pharmaceutical grade mFOLFIRINOX that was provided by The University of Texas MD Anderson Cancer Center pharmacy. Oxaliplatin was administered intravenously via the tail vein at $10 \mathrm{mg} / \mathrm{kg}$ in a volume of $0.01 \mathrm{~mL} / \mathrm{g}$. 5-FU (Fresenius Kabi, $50 \mathrm{mg} / \mathrm{mL}$ ) and Irinotecan (TEVA, $20 \mathrm{mg} / \mathrm{mL}$ ) were combined and diluted with $0.9 \%$ Sodium Chloride (normal saline) and given via intraperitoneal injection at $50 \mathrm{mg} / \mathrm{kg}$. Both injections account for one cycle delivered in the same day. For mice that received two cycles of mFOLFIRINOX, the second cycle was given 2 weeks after completion of the first cycle (Fig. 1).

For mice treated with gemcitabine/nab-paclitaxel, we used pharmaceutical grade gemcitabine $\left(\right.$ Gemzar $^{\circledR}$, NDC 00002-7501) and nab-paclitaxel (Abraxane ${ }^{\circledR}$, Celgene) generously provided by the Translational Research to AdvanCe Therapeutics and Innovation in ONcology (TRACTION). Gemcitabine was diluted with saline to $10 \mathrm{mg} / \mathrm{mL}$ and administered with intraperitoneal injections at $100 \mathrm{mg} / \mathrm{kg}$ in a volume of $0.01 \mathrm{~mL} / \mathrm{g}$, and given twice weekly. Nab-paclitaxel (Abraxane ${ }^{\circledR}$, Celgene) was diluted with saline to $30 \mathrm{mg} / \mathrm{mL}$ and administered intravenously at a dose of $300 \mathrm{mg} / \mathrm{kg}(30 \mathrm{mgPTX} / \mathrm{kg})$ in a volume of $0.01 \mathrm{~mL} / \mathrm{g}$, and given only once weekly one hour after one of the gemcitabine doses [14] (Fig. 1). 


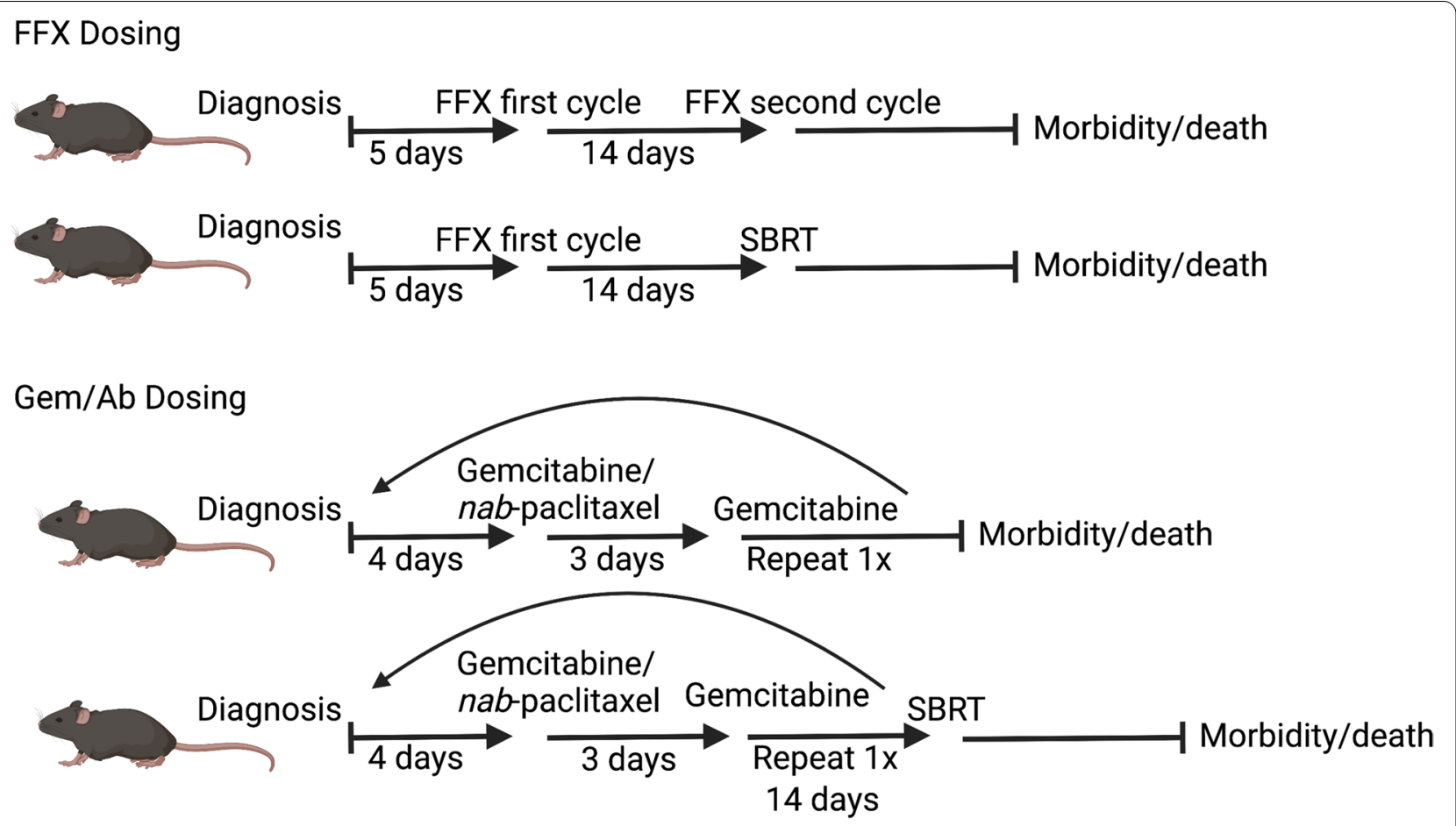

Fig. 1 Treatment dose scheme

\section{Chemotherapy dose finding and efficacy}

Mice with detectable tumors were randomized to receive modified FOLFIRINOX (mFFX) or gemcitabine/nabpaclitaxel (GEM/AB), and mice received therapy within $48 \mathrm{~h}$ of assignment. Both $\mathrm{mFFX}$ and GEM/AB arms were initially assigned to received one cycle of chemotherapy $(\mathrm{FFXx} 1$ and $\mathrm{GEM} / \mathrm{AB} \times 1)$. If no excessive toxicity was observed, then the next batch of mice would receive 2 cycles of chemotherapy (FFXx1 and GEM/AB $\times 1$ ). No further treatment was given after the last assigned dose of chemotherapy. Body weights were measured daily, and tumor growth monitored twice weekly with ultrasound. Endpoints were reached when mice met predetermined euthanasia endpoints, including tumors measuring greater than $15 \mathrm{~mm}$ in diameter, lethargy, weight loss exceeding $20 \%$ from baseline, or appearing moribund. At euthanasia, necropsies were conducted to look for macrometastases and determine a potential cause of death, as we have previously described [13].

\section{Radiation therapy}

For mice that received local treatment with radiation therapy, stereotactic body radiation therapy (SBRT) was delivered using an XRAD $225 \mathrm{Cx}$ irradiator with isoflurane anesthesia manifold and image guidance. The tumors were palpated and their locations marked with metallic beads. Then, radiation fields were aligned for each mouse using cone beam CT image guidance. The metallic beads were removed before radiation. Beam arrangement was anteroposterior/posteroanterior using a $10 \mathrm{~mm}$ diameter collimator, and mice received 5 fractions of $8 \mathrm{~Gy}$ with $24 \mathrm{~h}$ between fractions, for a total of 40 Gy which is a clinically accepted dose of radiation for pancreatic tumors [13]. mFOLFIRINOX SBRT mice were irradiated two weeks following one dose of chemotherapy. Gemcitabine/nab-paclitaxel SBRT mice were given two doses of chemotherapy and then received SBRT two weeks after the final dose. Mice were allowed to feed and drink water ad libitum throughout the duration of treatments. Additional supportive measures, such as moistened food pellets on the ground, were given if the mouse appeared dehydrated. Mice were also observed for radiation toxicity daily. The main symptoms assessed were the development of ruffled fur, persistent diarrhea, and/or drastic weight loss of $20 \%$ or more.

\section{Immunohistochemistry}

Thirty-seven tissue samples from murine pancreatic tumors were fixed in 10\% neutral buffered formalin and received for histopathology. Tissues were processed routinely, embedded in paraffin, sectioned at $4 \mathrm{~mm}$, and stained with H\&E. Five additional unstained sections from each tumor were submitted for immunohistochemical staining with rabbit monoclonal 
antibodies for pan-macrophage marker IBA-1 (Abcam, clone EPR16589, 1:8,000), and $\mathrm{T}$ cell markers: CD3 (Abcam clone SP7, 1:500), CD8 (Cell Signaling Technologies, clone D4W27, 1:400), CD4 (Cell Signaling Technologies, clone D7DZZ, 1:400), and FOXP3 (Cell Signaling Technologies, clone D608R, 1:200) using a Leica Bond Rx autostainer. Whole slide imaging was performed using Leica Biosystems Aperio AT2 digital slide scanner. Digital slides were viewed and representative images were captured at $20 \times$ magnification using Aperio ImageScope Software v12.4.3.7001. Each biomarker was quantified via digital image analysis using tuned algorithms in Leica Image Analysis Software in eSlide manager Spectrum Version 12.4.3.5008. Quantitative data was exported into an excel file and analyzed in GraphPad Prism Software Version 9 via one-way ANOVA; $p$ value $<0.05$ was considered significant.

\section{Statistical analysis}

Descriptive statistics were used to assess the baseline characteristics of mice and tumors in each group of mice. Continuous variables were presented as median and corresponding interquartile range (IQR). Categorical variables were presented as frequencies and percentages. We further assessed the average weight of mice in each group every day post-treatment. Changes over time in the weight of mice were plotted. Furthermore, survival curves for Overall Survival (OS) were generated using the Kaplan-Meier method, and log-rank $P$ values were calculated. OS was calculated from time of diagnosis until death of mice. Local tumor recurrence, distant metastasis, and cause of death were noted for all mice. Differences in local recurrence were assessed using the Chi-Square method. Statistical significance was set a priori at $P<0.05$. All statistical analyses were performed using IBM SPSS version 26. Kaplan-Meier curves were generated using Prism version 9. For immunohistochemistry analyses, treatment cohorts were compared using one-way ANOVA and individual pairwise comparisons with assessed with Student's t-test using Prism version 9.

\section{Results}

\section{Baseline mice and tumor characteristics}

In total, 92 mice were included and analyzed in our study. In particular, 54 mice were treated with chemotherapy only: 6 mice were treated with one cycle of mFOLFIRINOX (FFX X1), 30 mice with two cycles of mFOLFIRINOX (FFX X2), 6 mice with one cycle of gemcitabine/nab-paclitaxel (GEM/AB X1), 6 mice with two cycles of gemcitabine/nab-paclitaxel (GEM/AB X2), and 28 mice received only saline (control).

Table 1 presents the baseline characteristics of mice and tumors in mice treated with chemotherapy only. Mice in the FFX X2 group had the oldest age at diagnosis (24.8 weeks, IQR [21.7-26.9]), and those in the GEM/AB $\mathrm{X} 2$ had the youngest age at diagnosis (14.3 weeks, IQR [9.1-23.5]). A similar proportion of males and females were present in the FFX groups, and a few more males than females were present in the GEM/AB and control groups. Baseline weights were similar between all cohorts (between median: $23.6 \mathrm{~g}$, IQR 20.5-28.1 and median: $25.3 \mathrm{~g}$, IQR 22.0-28.4).

The baseline tumor diameter was largest in the FFX X1 group (median: $9.3 \mathrm{mmm}$ IQR 7.1-10.2), smallest in the GEM/AB X2 group (median: $4.8 \mathrm{~mm}$, IQR 4.0-6.9), and similar in the other 3 groups (between median: $6.7 \mathrm{~mm}$, IQR 6.2-7.4 and median: 7.1 mm, IQR 5.5-8.0). Most tumors were located in the body of the pancreas for the FFX X1 group $(3 / 6,50.0 \%)$, the tail of the pancreas for the FFX X2 group (13/30, 43.3\%), the head of the pancreas for the GEM/AB X1 group (4/6, 66.7\%), the tail of the pancreas for the GEM/AB X2 group (3/6, 50.0\%), and the

Table 1 Baseline mice and tumor characteristics in mice receiving chemotherapy only

\begin{tabular}{|c|c|c|c|c|c|}
\hline & FFX X1 $(n=6)$ & FFX X2 $(n=30)$ & GEM/AB X1 $(n=6)$ & GEM/AB X2 $(n=6)$ & Control $(n=28)$ \\
\hline Age & $16.9(10.9-26.1)$ & $24.8(21.7-26.9)$ & $21.3(16.1-25.2)$ & $14.3(9.1-23.5)$ & $21.7(17.4-25.6)$ \\
\hline Tumor Diameter at Dx & $9.3(7.1-10.2)$ & $6.7(6.2-7.4)$ & $7.1(5.5-8.0)$ & $4.8(4.0-6.9)$ & $7.0(5.0-8.0)$ \\
\hline Weight at Dx & $23.6(20.5-28.1)$ & $25.2(23.4-26.8)$ & $25.3(22.0-28.4)$ & $24.5(21.1-28.9)$ & $24.3(23.2-25.7)$ \\
\hline \multicolumn{6}{|l|}{ Sex } \\
\hline Female & $3(50.0)$ & $15(50.0)$ & $2(33.3)$ & $2(33.3)$ & $10(35.7)$ \\
\hline Male & $3(50.0)$ & $15(50.0)$ & $4(66.7)$ & $4(66.7)$ & $18(64.3)$ \\
\hline \multicolumn{6}{|l|}{ Tumor Location } \\
\hline Head & $0(0)$ & $7(23.3)$ & $4(66.7)$ & $2(33.3)$ & $13(46.4)$ \\
\hline Body & $3(50.0)$ & $10(33.3)$ & $2(33.3)$ & $1(16.7)$ & $10(35.7)$ \\
\hline Tail & $2(33.3)$ & $13(43.3)$ & $0(0)$ & $3(50.0)$ & $5(17.9)$ \\
\hline Neck & $1(16.7)$ & $0(0)$ & $0(0)$ & $0(0)$ & $0(0)$ \\
\hline
\end{tabular}


head of the pancreas for the control group (13/28, 46.4\%) (Table 1).

\section{Comparative analysis between mFOLFIRINOX} and gemcitabine/nab-paclitaxel reveals similar survival We ultimately wanted to treat KPC animals with at least two cycles of chemotherapy, to better approximate the multicycle regimens given to patients [7]. Thirty-six mice were treated with mFOLFIRINOX (6 mice with one cycle and 30 mice with two cycles). The median OS in the FFX X2 group was slightly longer that the median OS in the FFX X1 group (15 days vs 11 days, log-rank $P=0.003$ ) (Fig. 2A). Twelve mice were treated with gemcitabine/nab-paclitaxel (6 mice with one cycle and 6 mice with two cycles). Mice in the GEM/AB X2 group had longer OS when compared to mice in the GEM/AB $\mathrm{X} 1$ group (median OS, GEM/AB X2: 33.5 days vs GEM/ AB X1: 13 days, log-rank $P=0.001$ ) (Fig. 2B).

We compared OS between the mFFX and the GEM/AB treated mice. In mice that received one cycle of chemotherapy, the GEM/AB group had a slightly longer median OS compared to the FFX group, but this difference was not statistically significant (GEM/AB X1: 13 days, FFX $\mathrm{X} 1$ : 11 days, $\log$-rank $P=0.104$ ) (Fig. $3 \mathrm{~A}$ ). In mice that received two cycles of chemotherapy, the GEM/AB mice had better OS than the FFX mice (median OS, GEM/ AB X2: 33.5 days, FFX X2: 15 days, log-rank $P=0.002$ ) (Fig. 3B). All mice treated with chemotherapy showed a better median OS compared to the control group (median OS: 10.0 days, log-rank $P<0.001$ ) (Fig. 3C). Overall, in mice treated with chemotherapy, the GEM/ $\mathrm{AB} X 2$ group showed the best OS, and the FFX X1 group had the poorest results (log-rank $P<0.001)$ (Fig. 3C).
Our KPC animals are a validated model of autochthonous and localized pancreatic cancer. Thus, not surprisingly, local progression was the main cause of death in both FFX groups (FFX X1: 5, 83.3\%; FFX X2: 20, $66.7 \%, P=0.42$, Table 2), compared to distant metastasis (FFX X1: 1, 16.7\%; FFX X2: $11,36.7 \%, P=0.34$, Table 2). A similar pattern emerged in GEM/AB treated mice, where local progression of disease led to death more commonly than distant metastases (Table 2). Particularly, in the GEM/AB X1 cohort, 4 (66.7\%) mice died from local recurrence, and 1 (16.7\%) mouse died from distant metastasis. Moreover, in the GEM/AB X2 cohort 4 (66.7\%) mice died from local recurrence, and 2 (33.3\%) died from distant metastasis. In fact, ascites was observed in $7(7 / 36,14.4 \%)$ of $\mathrm{mFFX}$ and $1(1 / 12,8.3 \%)$ of GEM/AB mice.

We noted no major differences in acute toxicity between the treatment regimens, as assessed by weight changes (Fig. 4). The average weight of mice in the GEM/ $A B X 1$ and FFX $X 1$ decreased over time, while that of the GEM/AB X2 and FFX X2 increased.

\section{Chemotherapy induces changes in immune composition of the tumor microenvironment}

We performed immunohistochemistry on tumors collected during necropsies to assess relative numbers of immune infiltrates as a function of their treatment. Overall, macrophages (IBA-1+, Fig. 5A) predominated over $\mathrm{T}$ cells $(\mathrm{CD} 3+$, Fig. $5 \mathrm{~B})$ in the TME in all groups. There was a significant reduction in the number of macrophages and $\mathrm{T}$ cells in general in both groups treated with chemotherapy in comparison with the vehicle treated group. Further quantitative analysis of $\mathrm{T}$ cell subsets showed a trend

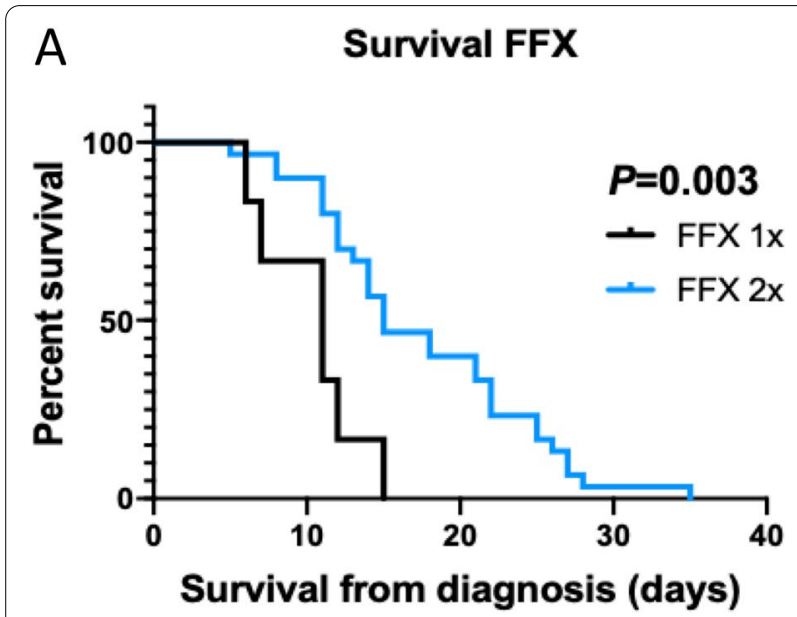

B Survival GEM/AB

Fig. 2 Kaplan-Meier Curve for Overall Survival in Mice Treated with One or Two Cycles of mFOLFIRINOX (FFX) A, and in Mice Treated with One or Two Cycles of gemcitabine/nab-paclitaxel (GEM/AB) B 

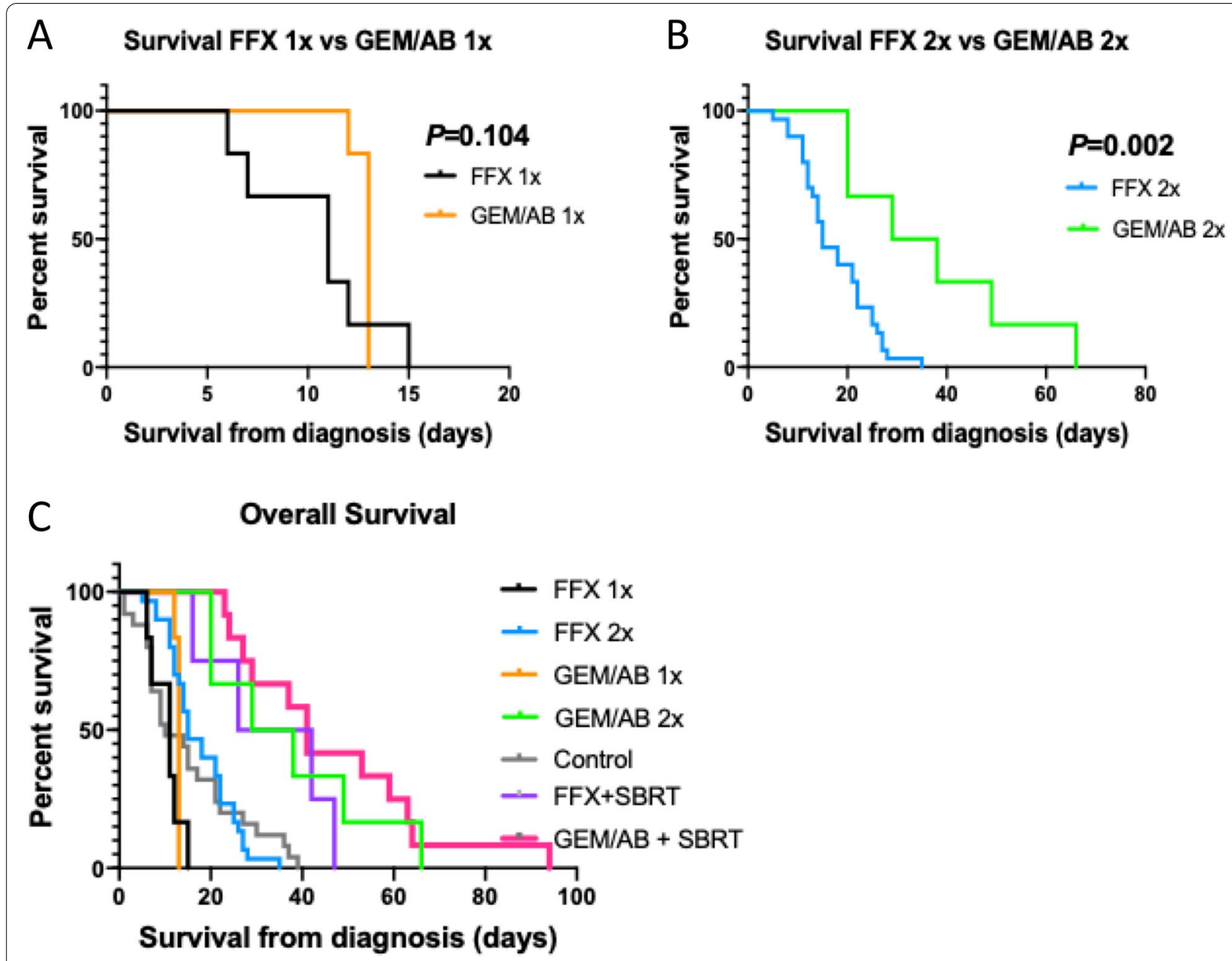

Fig. 3 Kaplan-Meier Curve for Overall Survival in Mice Treated with One Cycle of mFOLFIRINOX (FFX) or gemcitabine/nab-paclitaxel (GEM/AB) A, and in Mice Treated with Two Cycles of mFOLFIRINOX (FFX) or gemcitabine/nab-paclitaxel (GEM/AB) B, and Among All Mice $\mathbf{C}$

Table 2 Sites of recurrence/progression and cause of death in mice receiving chemotherapy only

\begin{tabular}{|c|c|c|c|c|c|}
\hline Recurrence/Progression & $\operatorname{FFXX1}(n=6)$ & FFX X2 $(n=30)$ & GEM/AB X1 $(n=6)$ & GEM/AB X2 $(n=6)$ & Control $(n=28)$ \\
\hline Local Recurrence & $5(83.3)$ & $20(66.7)$ & $4(66.7)$ & $4(66.7)$ & $19(67.9)$ \\
\hline Distant Metastasis & $1(16.7)$ & $11(36.7)$ & $2(33.3)$ & $3(50.0)$ & $7(25.0)$ \\
\hline \multicolumn{6}{|l|}{ Cause of Death } \\
\hline Local Recurrence & $5(83.3)$ & $20(66.7)$ & $4(66.7)$ & $4(66.7)$ & $19(67.9)$ \\
\hline Distant Metastasis & $1(16.7)$ & $3(10.0)$ & $1(16.7)$ & $2(33.3)$ & $7(25.0)$ \\
\hline
\end{tabular}

towards reduced cytotoxic $\mathrm{T}$ cell (CD8+, Fig. 5C) infiltration in the chemotherapy treated groups; however, CD8 $+\mathrm{T}$ cell preservation in the TME of the $\mathrm{Gem} / \mathrm{Ab}$ group appeared to be superior to tumors from FFX treated mice. There was a trend towards reduced regulatory $\mathrm{T}$ cells $(\mathrm{FOXP3}+$, Fig. 5D) in both chemotherapy treated groups in comparison to vehicle. $\mathrm{CD} 4+\mathrm{T}$ cell infiltration was decreased in the FFX treated group in comparison to vehicle, but $\mathrm{CD} 4+\mathrm{T}$ cells were preserved in the Gem/Ab treated group, with levels significantly higher than those in the FFX arm (Fig. 5E). 


\section{Weight Change Post-Treatment}

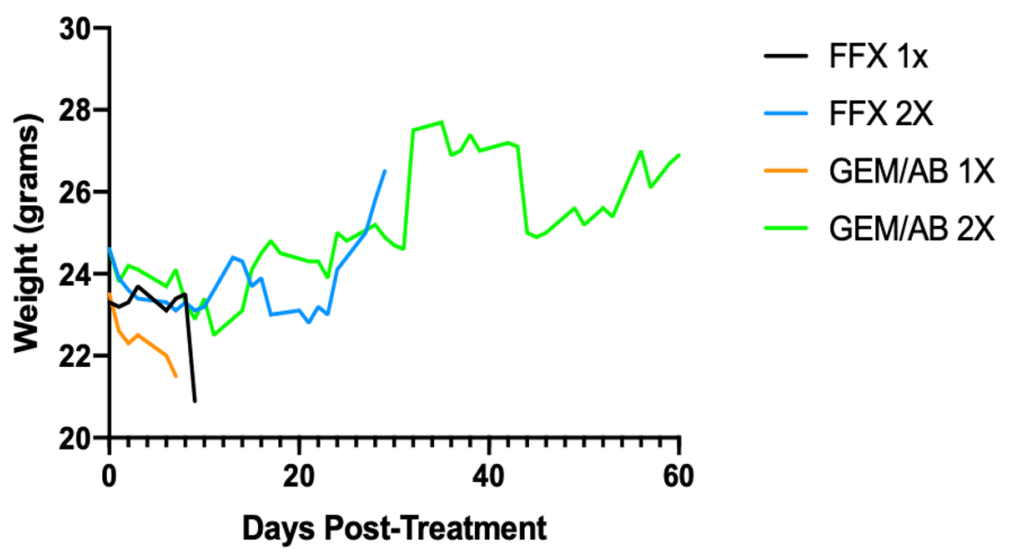

Fig. 4 Mice Weight Change After Chemotherapy Treatment

\section{Sequential chemotherapy followed by consolidative radiation may be superior to chemotherapy alone in KPC mouse models}

Radiation therapy is a commonly prescribed to consolidate treatment of the primary mass in pancreatic cancer, especially if surgery cannot be offered [15]. Stereotactic body radiation therapy (SBRT) has emerged as a convenient and powerful methods to achieve local control [16]. We previously demonstrated that SBRT is feasible in murine models [17]. To this end, we treated cohorts of KPC mice that first received mFOLFIRINOX or gemcitabine/nabpaclitaxel, then immediately followed with a clinically relevant dose of SBRT (8 Gy $\times 5$ fractions). Sixteen mice were treated with sequential chemoradiation: 4 mice (25\%) were treated with one cycle of mFOLFIRINOX and SBRT, and 12 mice (75\%) were treated with two cycles of gemcitabine/nab-paclitaxel and SBRT.

Table 3 presents the baseline characteristics of mice treated with consolidative SBRT after induction chemotherapy. The median OS in the FFX + SBRT group was 34 days, and the median OS in the GEM/AB + SBRT group was 41 days (logrank $P=0.214$ ) (Fig. 6). The addition of SBRT to both $\mathrm{mFFX}$ and GEM/AB regimens seems to show a trend towards improved survival compared to mice treated with chemotherapy only (Fig. 3). In total, 4 (25\%) mice treated with chemoradiation died from local recurrence: 3 in the FFX group $(3 / 4,75 \%)$, and 1 in the GEM/AB group (1/12, 8.3\%) (Table 4). Only one mouse $(8.3 \%)$ in the GEM/AB group died from radiation toxicity.

\section{Discussion}

The use of chemotherapy is common in patients with pancreatic cancer since most tumors present with advanced or metastatic disease where local therapy is of limited clinical benefit. Two common chemotherapy regimens in the modern treatment of pancreatic cancer are mFOLFIRINOX and gemcitabine/nab-paclitaxel [8, 9, 18, 19]. However, the use of those regimens is not routinely performed in pre-clinical models that often inform targets for clinical trials. In our study, we show that treatment with mFOLFIRINOX and gemcitabine/nab-paclitaxel is feasible and prolong survival. Thus, the routine use of clinically relevant chemotherapy should be considered in studies involving spontaneous pancreatic tumors in KPC mice.

When analyzing mice that were treated with chemotherapy only, we found that mice treated with two cycles of gemcitabine/nab-paclitaxel had the best survival outcomes (median OS: 33.5 days, Fig. 3C). There was no obvious immune biomarker to explain the biological differences between gemcitabine/nab-paclitaxel and mFFX, though we observed greater numbers of CD4 + cells in tumors after treatment with gemcitabine/nab-paclitaxel, compared to mFFX (Fig. 5E)). CD4+ cells can differentiate into subtypes that both serves as anti-tumor effectors (Th1 lineage) or suppressor cells $(\mathrm{CD} 4+\mathrm{CD} 25+)$ $[20,21]$. The relevance of these subtypes in the clinic are not clear. One study suggested that mFFX increases Th1 effectors [20], while others have another found that FFX or Gem/Ab induced similar immune changes, based on peripheral blood markers [22]. Thus, our observations serve as a benchmark for other preclinical and correlative translational studies, which may shed further light on the mechanisms behind these findings. 


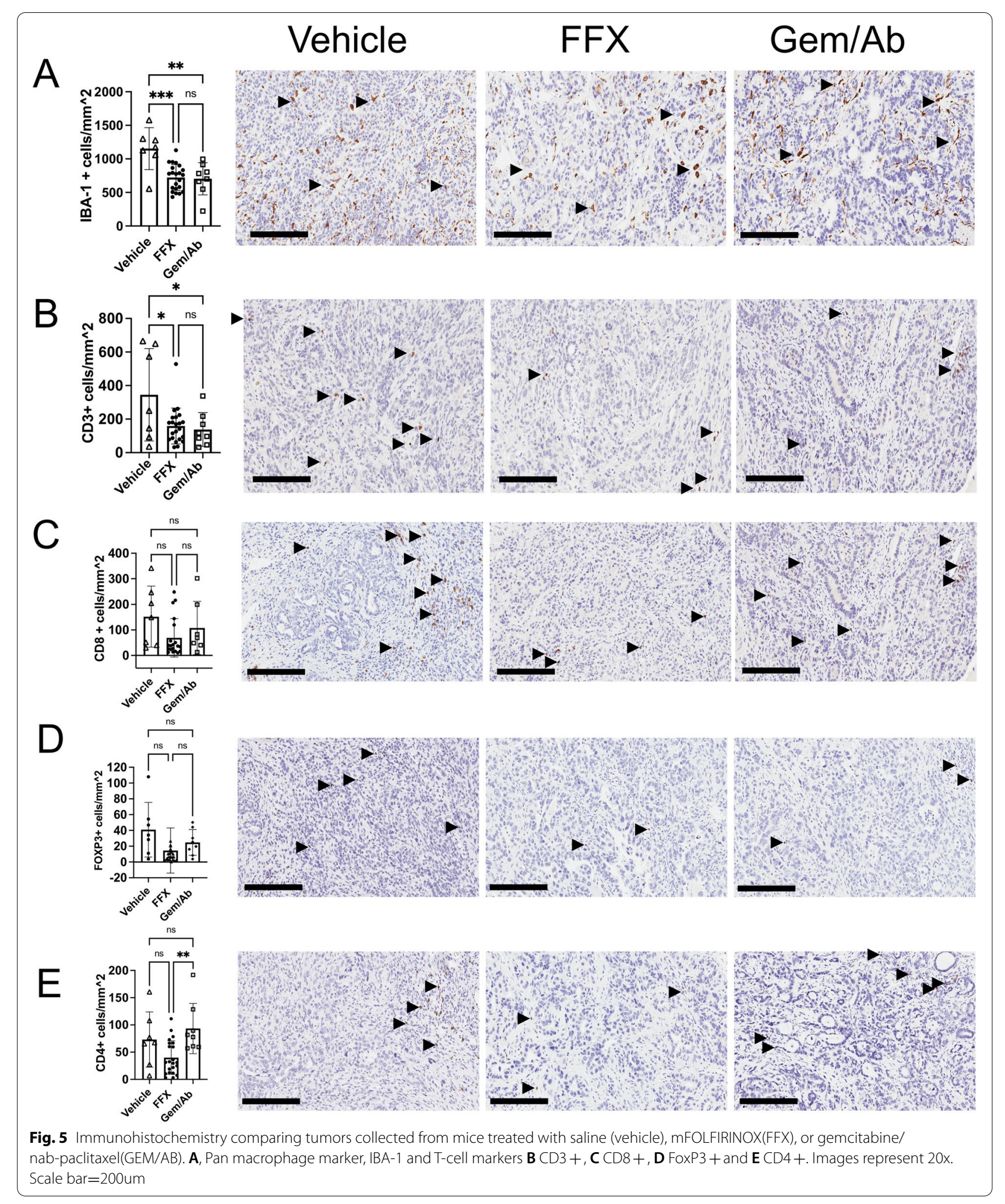


Table 3 Baseline mice and tumor characteristics in mice receiving chemoradiation

\begin{tabular}{lll}
\hline & FFX + SBRT $(\boldsymbol{n}=\mathbf{4})$ & GEM/AB+ SBRT $(\boldsymbol{n = 1 2})$ \\
\hline Age & $5.2(5.0-6.5)$ & $4.7(4.4-5.1)$ \\
Tumor Diameter at Dx & $5.6(4.5-6.5)$ & $5.2(4.36 .4)$ \\
Weight at Dx & $22.7(19.8-24.8)$ & $25.5(21.5-29.3)$ \\
Sex & & \\
$\quad$ Female & $3(75.0)$ & $4(33.3)$ \\
$\quad$ Male & $1(25.0)$ & $8(66.7)$ \\
Tumor Location & & \\
$\quad$ Head & $1(25.0)$ & $4(33.3)$ \\
$\quad$ Body & $2(50)$. & $6(50.0)$ \\
Tail & $1(25.0)$ & $2(16.7)$ \\
\hline
\end{tabular}

We also note another potentially confounding factor in these results in that a major component of this regimen is the nanoparticle-albumin-bound paclitaxel which contains albumin, a human derived protein [23]. As such, using $n a b /$ paclitaxel in KPC mice could be postulated to lead to significant toxicity due to cross reactivity with the human component of the drug with repeated doses. However, we show that gemcitabine/nab-paclitaxel was very well tolerated by KPC mice even with repeated dosing, and even led to improved OS compared to mFOLFIRINOX (Fig. 3A, 3B). Additionally, the improved survival associated with the use of $n a b /$ paclitaxel compared to mFOLFIRINOX could be related to lower toxicity, as has been shown in clinical studies [10]. This similar effect was observed in prior studies, but it is unclear whether prolonged administration of GEM/AB could be feasible.
Table 4 Cause of death in mice receiving chemoradiation

\begin{tabular}{lll}
\hline Cause of Death & FFX + SBRT $(\boldsymbol{n}=\mathbf{4})$ & $\begin{array}{l}\text { GEM/ } \\
\text { AB }+ \text { SBRT } \\
(\boldsymbol{n}=\mathbf{1 2})\end{array}$ \\
\hline Local Recurrence & $3(75.0)$ & $1(8.3)$ \\
Distant Metastasis & $1(25.0)$ & $5(41.7)$ \\
Radiation Toxicity & $0(0)$ & $1(8.3)$ \\
Unknown & $0(0)$ & $5(41.7)$ \\
\hline
\end{tabular}

When assessing weight change, mice treated with gemcitabine/nab-paclitaxel had similar weight change trends to those treated with mFOLFIRINOX (Fig. 4). Necropsy analysis revealed that mice treated with gemcitabine/nab-paclitaxel died because of disease progression, without significant treatment toxicity. Unfortunately, we did not collect normal tissues but expect that the toxicity mirrors that of clinical patients in that gemcitabine/nab paclitaxel is typically less toxic than $\mathrm{mFOL-}$ FIRINOX. We note that mice treated with two cycles of gemcitabine/nab-paclitaxel gained weight between diagnosis and death, which we attribute to abdominal ascites and tumor progression. Additionally, in that regard, it seems that gemcitabine/nab-paclitaxel could be safely considered in KPC mice with spontaneous pancreatic tumors.

Despite the improved outcomes in mice treated with gemcitabine/nab-paclitaxel, the mice cohorts treated with mFOLFIRINOX still showed reasonable OS (median OS, FFX X1: 11 days, FFX X2: 15 days) (Fig. 2A). Moreover, mice treated with one or two cycles of mFOLFIRINOX had longer OS compared to mice in the control cohort (Fig. 3C). As such, mFOLFIRINOX

\section{Survival SBRT}

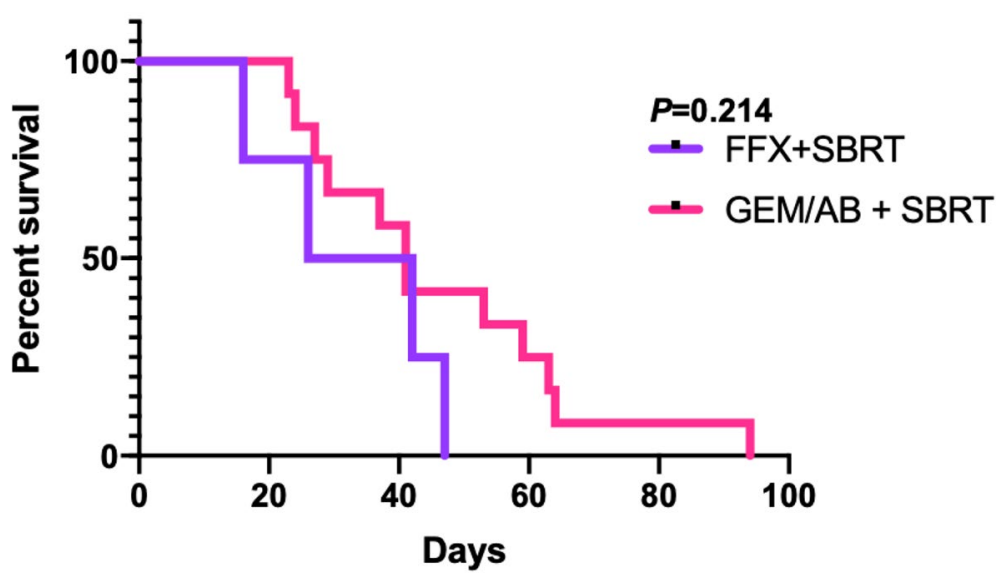

Fig. 6 Kaplan-Meier Curve for Overall Survival in Mice Treated Stereotactic Body Radiation Therapy (SBRT) and mFOLFIRINOX (FFX) or gemcitabine/nab-paclitaxel (GEM/AB) 
can also be safely considered in KPC mice. This feasibility study was not designed to assess potential futility of mFFX compared to gemcitabine/nab-paclitaxel, but we do note that the there was a survival trend in the latter combination with a more favorable toxicity profile, as has been reported in a recent Phase II study [24].

Lastly, we analyzed the use of chemoradiation with SBRT in KPC mice, and we show that the addition of local treatment with radiation therapy to chemotherapy further improved OS (Fig. 3C). This goes in line with pancreatic cancer treatment in humans, where local therapy with surgery and/or radiation therapy has been shown to improve survival and recurrence rates $[25,26]$. Furthermore, the addition of SBRT could have potentially increased the tumors' sensitivity to chemotherapy, and altered the immune tumor's immune environment, and by doing so, helped improve overall survival. It is important to note that as this is a preliminary study, we did not perform any sham radiation, and hence do not have a sham radiated control group. Nevertheless, KPC mice tolerated SBRT treatment well, with only one mouse dying of radiation-induced toxicity (Table 4). SBRT treatment has become increasingly common in clinical practice, and as such, incorporating chemoradiation with SBRT in pre-clinical models would help to best parallel clinical management. In that regard, our data show that mFOLFIRINOX, nab/paclitaxel, and SBRT can be safely used and combined in KPC mice.

\section{Conclusions}

Our data show that the use of mFOLFIRINOX and gemcitabine/nab-paclitaxel is feasible in spontaneous pancreatic tumors in KPC mouse models. Mice receiving two cycles of chemotherapy had improved survival, and did not show signs of severe toxicity. We further show that chemoradiation using mFOLFIRINOX or gemcitabine/nab-paclitaxel chemotherapy regimens with SBRT is also feasible, and was also well tolerated in KPC mice. We believe that our results show that the use of clinically relevant chemotherapy and chemoradiation regimens is feasible in KPC mouse models. As such, we encourage future pre-clinical studies to incorporate modern chemotherapy combinations in order to best mimic the current clinical standard of care.

\section{Abbreviations \\ FFX: FOLFIRINOX; GEM/AB: Gemcitabine/nab-paclitaxel; IQR: Interquartile range; mFFX: Modified FOLFIRINOX; OS: Overall survival; SAIF: Small Animal Imaging Facility; SBRT: Stereotactic body radiation therapy.}

\section{Acknowledgements}

We would like to thank the University of Texas MD Anderson Cancer Center Institute for Applied Cancer Science (IACS) for generously providing the chemotherapy used in our study. This research is supported in part by the National Institutes of Health through MD Anderson's Cancer Center Support Grant CA016672.

This work was also supported by the $\mathrm{NIH} / \mathrm{NCl}$ under award number P30CA016672 for use of the Small Animal Imaging Facility. Figures were made with BioRender.

\section{Authors' contributions}

$A M D, J A J$, and CMT were responsible for the concept and study study design. AMD performed all the mouse experiments and statistical analysis performed by JAJ. MMG and NWF performed all immunohistochemistry experiments. All authors contributed substantially to the writing of this manuscript, through performing literature review, data analysis, data interpretation, manuscript drafting, and providing significant comments and/or edits to the final manuscript. The authors read and approved the final manuscript.

\section{Funding}

CMT is supported by funding from NIH under award R01CA227517-01A1, Cancer Prevention \& Research Institute of Texas (CPRIT) grant RR140012,V Foundation (V2015-22), the Reaumond Family Foundation, the Childress Family Foundation, the Mark Foundation, and the McNair Foundation. CJGG was supported by funding from NIH under awards F31DK121384 and U54CA096300/297. This research is supported in part by the MD Anderson Cancer Center Support Grant CA016672 including animal housing and care in the Research Animal Support Facility (RASF).This work was also supported by the $\mathrm{NIH} / \mathrm{NCl}$ under award number P30CA016672 for use of the Small Animal Imaging Facility.

\section{Availability of data and materials}

Research data are stored in an institutional repository and will be shared upon reasonable request to the corresponding author.

Data is available upon request to the corresponding author.

\section{Declarations}

\section{Ethics approval and consent to participate}

The University of Texas MD Anderson Cancer Center Institutional Review Board approved all protocols in this study. All mouse work was approved and done in accordance with the Institutional Animal Care and Use Committee of The University of Texas MD Anderson Cancer Center under protocol IACUC \#00001252-RN02. The studies were carried out in compliance with ARRIVE guidelines.

\section{Consent for publication \\ Not applicable.}

\section{Competing interests}

Dr. CMT is on the medical advisory board of Accuray and is a paid consultant for Xerient Pharma and Phebra Pty, Ltd. All other authors report no competing interests.

\section{Author details}

${ }^{1}$ Department of Experimental Radiation Oncology, The University of Texas MD Anderson Cancer Center, Houston, TX 77030, USA. ² Department of Radiation Oncology, The University of Texas MD Anderson Cancer Center, Houston, TX 77030, USA. ${ }^{3}$ Department of Veterinary Medicine and Surgery, The University of Texas MD Anderson Cancer Center, Houston, TX 77030, USA. ${ }^{4}$ Translational Research To AdvanCe Therapeutics and Innovation in ONcology (TRACTION), The University of Texas MD Anderson Cancer Center, Houston, TX 77030, USA.

Received: 26 August 2021 Accepted: 27 January 2022

Published: 16 February 2022

\section{References}

1. Siegel RL, Miller KD, Fuchs HE, Jemal A. Cancer statistics, 2021. CA Cancer J Clin. 2021;71:7-33.

2. Rahib L, Smith BD, Aizenberg R, Rosenzweig AB, Fleshman JM, Matrisian LM. Projecting cancer incidence and deaths to 2030: the unexpected 
burden of thyroid, liver, and pancreas cancers in the united states. Cancer Res. 2014;74:2913-21.

3. Chakraborty S, Singh S. Surgical resection improves survival in pancreatic cancer patients without vascular invasion- a population based study. Ann Gastroenterol. 2013;26:346-52.

4. Bayne $\amalg$, Beatty GL, Jhala N, Clark CE, Rhim AD, Stanger BZ, et al. Tumorderived granulocyte-macrophage colony-stimulating factor regulates myeloid inflammation and T cell immunity in pancreatic cancer. Cancer Cell. 2012;21:822-35.

5. Oettle H, Post S, Neuhaus P, Gellert K, Langrehr J, Ridwelski K, et al. Adjuvant chemotherapy with gemcitabine vs observation in patients undergoing curative-intent resection of pancreatic cancer: a randomized controlled trial. JAMA. 2007;297:267-77.

6. Neoptolemos JP, Stocken DD, Friess H, Bassi C, Dunn JA, Hickey H, et al. A randomized trial of chemoradiotherapy and chemotherapy after resection of pancreatic cancer. N Engl J Med. 2004;350:1200-10.

7. Conroy T, Hammel P, Hebbar M, Ben Abdelghani M, Wei AC, Raoul J-L, et al. FOLFIRINOX or gemcitabine as adjuvant therapy for pancreatic cancer. N Engl J Med. 2018;379:2395-406.

8. Conroy T, Desseigne F, Ychou M, Bouché O, Guimbaud R, Bécouarn Y, et al. FOLFIRINOX versus gemcitabine for metastatic pancreatic cancer. N Engl J Med. 2011;364:1817-25.

9. Chun JW, Lee SH, Kim JS, Park N, Huh G, Cho IR, et al. Comparison between FOLFIRINOX and gemcitabine plus nab-paclitaxel including sequential treatment for metastatic pancreatic cancer: a propensity score matching approach. BMC Cancer. 2021;21:537.

10. Riedl JM, Posch F, Horvath L, Gantschnigg A, Renneberg F, Schwarzenbacher E, et al. Gemcitabine/nab-Paclitaxel versus FOLFIRINOX for palliative first-line treatment of advanced pancreatic cancer: A propensity score analysis. Eur J Cancer. 2021;151:3-13.

11. Aguirre AJ, Bardeesy N, Sinha M, Lopez L, Tuveson DA, Horner J, et al. Activated Kras and Ink4a/Arf deficiency cooperate to produce metastatic pancreatic ductal adenocarcinoma. Genes Dev. 2003;17:3112-26.

12. Yang Y, Armour M, Wang KK-H, Gandhi N, lordachita I, Siewerdsen J, et al. Evaluation of a Cone Beam Computed Tomography Geometry for Image Guided Small Animal Irradiation. Phys Med Biol. 2015;60:5163-77.

13. Fujimoto TN, Colbert LE, Huang Y, Molkentine JM, Deorukhkar A, Baseler $L$, et al. Selective EGLN inhibition enables ablative radiotherapy and improves survival in unresectable pancreatic cancer. Cancer Res. 2019:79:2327-38

14. Frese KK, Neesse A, Cook N, Bapiro TE, Lolkema MP, Jodrell DI, et al. nab-paclitaxel potentiates gemcitabine activity by reducing cytidine deaminase levels in a mouse model of pancreatic cancer. Cancer Discov. 2012;2:260-9.

15. Abi Jaoude J, Kouzy R, Nguyen ND, Lin D, Noticewala SS, Ludmir EB, et al. Radiation therapy for patients with locally advanced pancreatic cancer: Evolving techniques and treatment strategies. Curr Probl Cancer. 2020;44(60):100607.

16. Abi Jaoude J, Thunshelle CP, Kouzy R, Nguyen ND, Lin D, Prakash L, et al. Stereotactic versus conventional radiation therapy for patients with pancreatic cancer in the modern era. Adv Radiat Oncol. 2021;6(6):100763.

17. Molkentine JM, Fujimoto TN, Horvath TD, Grossberg AJ, Garcia CJG, Deorukhkar A, et al. Enteral Activation of WR-2721 Mediates Radioprotection and Improved Survival from Lethal Fractionated Radiation. Sci Rep. 2019:9:1949.

18. Goldstein D, El-Maraghi RH, Hammel P, Heinemann V, Kunzmann V, Sastre J, et al. nab-Paclitaxel plus gemcitabine for metastatic pancreatic cancer: long-term survival from a phase III trial. J Natl Cancer Inst. 2015;107(2):dju413.

19. Blomstrand H, Scheibling U, Bratthäll C, Green H, Elander NO. Real world evidence on gemcitabine and nab-paclitaxel combination chemotherapy in advanced pancreatic cancer. BMC Cancer. 2019;19:40.

20. Peng H, James CA, Cullinan DR, Hogg GD, Mudd JL, Zuo C, et al. Neoadjuvant FOLFIRINOX therapy is associated with increased effector t cells and reduced suppressor cells in patients with pancreatic cancer. Clin Cancer Res. 2021;27(24):6761-71. https://doi.org/10.1158/1078-0432. CCR-21-0998.

21. Hinz S, Pagerols-Raluy L, Oberg H-H, Ammerpohl O, Grüssel S, Sipos B, et al. Foxp3 expression in pancreatic carcinoma cells as a novel mechanism of immune evasion in cancer. Cancer Res. 2007;67:8344-50.
22. Sams $L$, Kruger $S$, Heinemann V, Bararia D, Haebe $S$, Alig S, et al. Alterations in regulatory $T$ cells and immune checkpoint molecules in pancreatic cancer patients receiving FOLFIRINOX or gemcitabine plus nab-paclitaxel. Clin Transl Oncol. 2021;23:2394-401.

23. Giordano G, Pancione M, Olivieri N, Parcesepe P, Velocci M, Di Raimo T, et al. Nano albumin bound-paclitaxel in pancreatic cancer: Current evidences and future directions. World J Gastroenterol. 2017;23:5875-86.

24. Ozaka M, Ueno M, Ishii H, Mizusawa J, Katayama H, Kataoka T, et al. Randomized phase II study of modified FOLFIRINOX versus gemcitabine plus nab-paclitaxel combination therapy for locally advanced pancreatic cancer (JCOG1407). JCO. 2021;39(15):4017-4017.

25. Katz MHG, Ou FS, Herman JM, Ahmad SA, Wolpin B, Marsh R, et al. Alliance for clinical trials in oncology (ALLIANCE) trial A021501: preoperative extended chemotherapy vs. chemotherapy plus hypofractionated radiation therapy for borderline resectable adenocarcinoma of the head of the pancreas. BMC Cancer. 2017;17:505.

26. Koong AC, Le QT, Ho A, Fong B, Fisher G, Cho C, et al. Phase I study of stereotactic radiosurgery in patients with locally advanced pancreatic cancer. Int J Radiat Oncol Biol Phys. 2004;58:1017-21.

\section{Publisher's Note}

Springer Nature remains neutral with regard to jurisdictional claims in published maps and institutional affiliations.

Ready to submit your research? Choose BMC and benefit from:

- fast, convenient online submission

- thorough peer review by experienced researchers in your field

- rapid publication on acceptance

- support for research data, including large and complex data types

- gold Open Access which fosters wider collaboration and increased citations

- maximum visibility for your research: over 100M website views per year

At BMC, research is always in progress.

Learn more biomedcentral.com/submissions 